\title{
Magnetic Nanoliposomes: A RevieW on Preparation AND Characterization MethodS
}

\author{
Palanisamy Selvamani*, Venkata Krishnan Pari, Rosi Rajamanickam and Subbiah Latha \\ Department of Pharmaceutical Technology, University College of Engineering, Anna University, \\ BIT Campus, Tiruchirappalli-620024, Tamil Nadu, India
}

*E-mail: selvamani@aubit.edu.in

Tel.: +919842478785.

Received: Mar 13, 2021 / Revised: Jun 06, 2021 / Accepted: Jun 07, 2021

\begin{abstract}
Magnetic nanoliposomes are versatile nanocarriers for targeted drug delivery. These liposomes can enhance the efficacy of bioactive compounds by improving pharmacokinetic parameters. Chemical ingredients of magnetic nanoliposomes include ferrofluid, cholesterol and phospholipid molecules. The preparation of magnetic nanoliposomes involves two steps viz. synthesis of magnetic nanoparticles and then combination with prepared nanoliposomes. The magnetic nanoliposomes are synthesized by various chemical methods including co-precipitation, thermal decomposition, microemulsion and hydrothermal synthesis. The nanoliposomes are prepared by sonication, extrusion, micro-fluidization techniques. Characterization studies included size, nanoparticle tracking analysis, flow cytometry, size exclusion chromatography, DSC, encapsulation efficiency. This study provides an overview of the preparation and characterization techniques of magnetic nanoliposomes.
\end{abstract}

Key words: Magnetic nanoliposome, Ferrofluid, Co-precipitation, Sonication, Flow cytometry.

\section{INTRODUCTION}

Magnetic nanoliposomes are the most preferably investigated nano carriers for targeted drug delivery. Stabilization of therapeutic compounds helps to overcome barriers associated with Absorption in tissue and cellular level, and it can optimize the bio-distribution parameters and therapeutic efficiency can be increased in various drug delivery applications [1].

The synthetic bilayered structures can be biocompatible and range up to seven hundred nanometers in size. In addition, these nanostructures are capable of enclosing both hydrophobic and hydrophilic drugs, enabling their protection from the external inactivating conditions [2]. The liposomes are the small and spherical vesicles generated from phospholipids and cholesterol [3]. Typical range diameter of Liposomes for drug delivery is from $250 \mathrm{~A}^{\circ}$ to several micrometers. Advantages and limitations of liposomes depends on the physical, chemical and biological characteristics such as size, loading capacity, encapsulation efficiency and stability parameters. Research in liposomal drug delivery is developed, and multiple reviews of the research have been composed [4]. With recent developments in liposome formulations and their applications in medicine, this analysis aims to provide a detailed insight into magnetic nanoliposome technology [5].

\section{Nanoliposomes}

Nanoliposomes developed recently refers to nanoscale lipid vesicles. The formulation, properties and mechanism of nanoliposomes resemble liposome. The surface area of liposomes is probably high and it can have potential to enhance solubility and bioavailability. The controlled release from the encapsulated form of liposomes is increased [3]. They have various applications in fields of 
cancer therapy, nutraceuticals and cosmetics because of technological advancements. It can alter the pharmacokinetic parameters and increase the efficacy of bioactive compounds [6]. Despite the enormous research and development works on nanoliposomal technology, only a few products have been approved for human use, and many products are in clinical trials. This may be due to identified toxicity studies in liposomal formulations, stability issues, and high cost of production [7]. Incorporation of hydrophilic drugs in inner layer of phospholipid and hydrophobic drugs in phospholipid bilayer. Nanoliposomes plays a crucial role in targeted drug delivery system and has numerous advantages over conventional drug delivery. It can also be used for passive targeting or active targeting of drug components [3] (Figure 1).

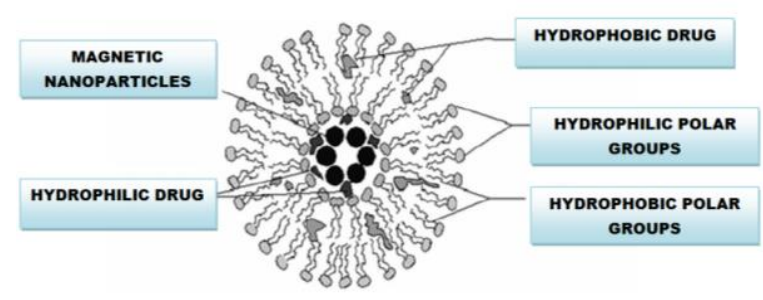

Fig. 1. Structure of magnetic nanoliposome

\section{Chemical constituent of magnetic nano- liposomes}

The chief chemical ingredients of magnetic nanoliposomes are ferrofluid, cholesterol and phospholipids [8]. Because of amphiphilic nature, they contain both hydrophilic and hydrophobic properties. In phospholipid, head group is hydrophilic and its fatty acid tail is hydrophobic. Sterol incorporation into liposomes brought major changes in vesicle properties. Cholesterol is mostly used in liposomes manufacturing. It can enhance the liposomal structure by modifying the fluidity of lipoidal membrane and prevents crystallization [9]. It is used to stabilize the nanoliposomes and permeability to a lipid membrane. It leads to the stability of nanoliposomes and decreases lipid membrane permeability. Magnetic liposomes comprise magnetic nanoparticles prepared by various chemical methods. The magnetic particles in ferrofluid are magnetite, $\mathrm{Fe}_{3} \mathrm{O}_{4}$, although other magnetic particles have been used $[10,11]$.

\section{Synthesis of magnetic nanoliposomes}

Synthesis of magnetic nanoparticles
Bottom up and top-down method are the two principal methods used in preparing magnetic nanoparticles, mostly, the bottom-up method is the most preferred, because of particle size limitations of top-down method.

The bottom-up method can be co-precipitation, thermal decomposition, reverse micelle, and hydrothermal etc. The most commonly employed methods are co-precipitation and hydrothermal synthesis [12].

\section{Co-precipitation method}

The co-precipitation synthesis procedure is a very simple method for preparing MNP. Most research work uses aqueous media for precipitation. Very often, the magnetic iron oxides are prepared by a co-precipitation from aqueous $\mathrm{Fe}^{2+}$ and $\mathrm{Fe}^{3+}$ salt solutions, to which a base is added [13,14]. There are also some disadvantages with this method. Controlling the $\mathrm{pH}$ is vital in order to control the particle size, which is governed by kinetic factors.

\section{Thermal decomposition method}

The thermal decomposition of organometallic in boiling organic solvents is another effective way for Magnetic nanoparticle preparation. The synthesized MNP shows a narrow size distribution. Mostly Iron carbonyls/iron acetylacetonates non-magnetic precursors for the synthesis [15]. By mild heating of these metal particles under oxidative conditions, it is oxidized to iron oxide. A single step synthesis involves thermal decomposition of precursors with cation iron centers.

\section{Micro-emulsion synthesis}

Micro-emulsion synthesis is a two-phase method for the synthesis of monodisperse Magnetic nanoparticles. For this purpose, a water-in-oil micro-emulsion is prepared by dispersion of nanosized water droplets $(10-50 \mathrm{~nm})$ in an oil phase, stabilized by surfactant molecules at the water/oil interface [15]. The properties of NPs prepared by the micro-emulsion method depend on the type and structure of the surfactant [16]. MNP of metallic cobalt, cobalt/platinum alloys, and gold-coated cobalt/platinum have been synthesized by this method [17]. It shows narrow size distribution, which will be useful for the medical applications [18].

\section{Hydrothermal synthesis}

This is basically a solution reaction-based approach. Formation of nanomaterials can 
happen in a wide temperature range from room temperature to very high temperatures. Hydrothermal synthesis performed in aqueous media at temperatures above $200{ }^{\circ} \mathrm{C}$ is realized in autoclaves at pressures above 2000 psi [15]. This route exploits the ability of water to hydrolyze and dehydrate metal salts at high temperatures [19]. Because of the low solubility of the obtained metal oxide particles in water at such temperatures, a precipitation takes place and by variation of concentration, temperature, and autoclaving time, particle size and morphology can be controlled [19] (Figure 2).

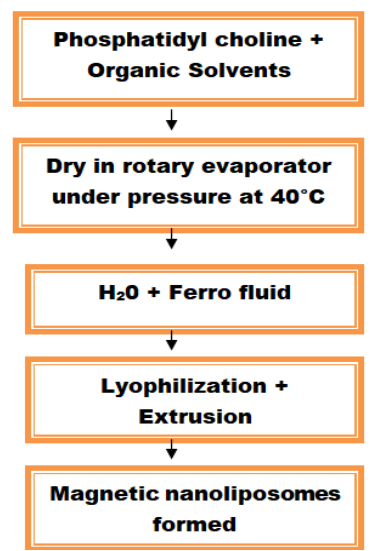

Fig. 2. Synthetic scheme for magnetic nanoliposomes

\section{Methods of liposome preparation}

Figure 3 enlists diverse methods of liposomes preparation.

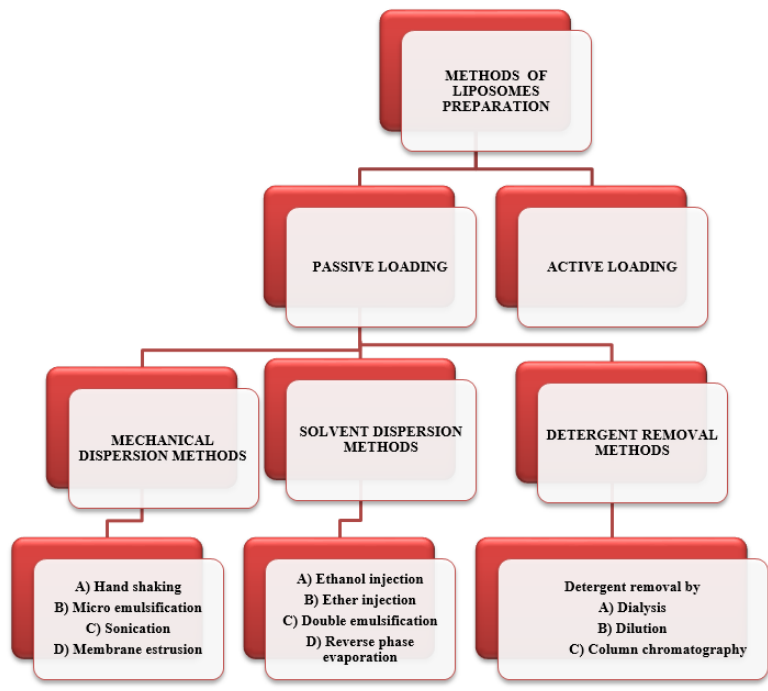

Fig. 3. Methods of liposomes preparation

\section{Preparation of nanoliposomes}

\section{Sonication method}

Sonication is one of the most widely used method to prepare nanoliposomes of small size ranges [20] Titanium coated probe sonicator used for treatment of hydrated vesicles. The procedure involves dissolving an appropriate composition of phospholipids and cholesterol in a mixture of chloroform and methanol. Filtering of mixture are carried out to remove insoluble components. The filtrate is transferred to round bottom flasks runs in rotary evaporator to remove organic solvents. After evaporation of organic solvents allow to stand vacuum overnight at $0.1 \mathrm{pa}$. Suitable buffer is added to hydrate lipid vesicles from dry lipid film. After hydration, multilamellar vesicles are formed. With sonication with probe sonicator, unilamellar vesicles are produced. Probe sonication is preferred over bath sonication because energy input is high. Probe sonicator has a disadvantage that it can release titanium particles to lipid dispersion [21] (Figure 4).

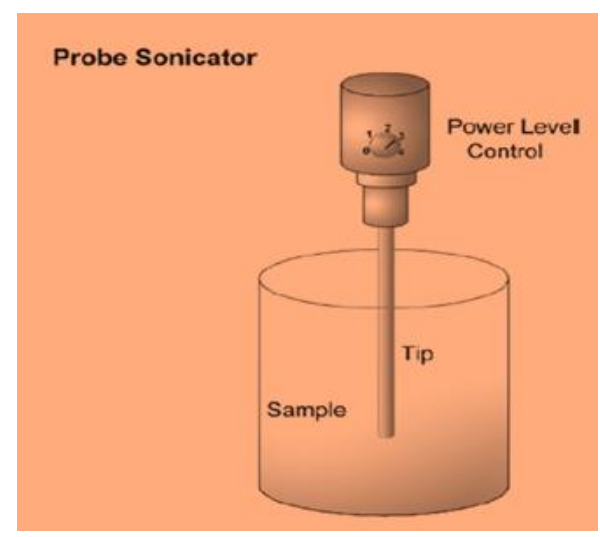

Fig. 4. Nanoliposomes preparation by Sonication

\section{Extrusion method}

In extrusion technique, the liposomal suspension is passed through a membrane filter. Extruder is a machine equipped with a pump which pushes fluids through the membrane filter. In this process, multilamellar large vesicles (MLVs) are changed into large unilamellar vesicles (LUVs) with the use of desired pore size of filters [3]. Parameters such as pore size, pressure applied pressure and number of cycles influence the diameter and size distribution of the formed liposomes. Extruder is cleaned by rinsing with ethanol. To prepare MLV liposomes, two stacked polycarbonate filters are placed in a stainless steel filter holder of extruder, followed by loading of the liposome suspension into one syringe of mini extruder. Second syringe is to be placed at another end of extruder. The temperature is allowed to reach heating block and the suspension is passed through extruder. Repeat extrusion process for seven times [22]. 


\section{Micro-fluidization technique}

Micro-fluidization method of nanoliposome formation precludes the use of toxic chemicals using micro-fluidizer. It is a new diffusion-drive technique to reduce vesicle size employing the force of two streams of liposome suspension that collide under high pressure [7].

Ingredients for preparation of nanoliposomes and suspension medium are selected based on intended application. Phospholipid dispersion is prepared using homogenizer and the dispersion formed is passed through micro-fluidizer. The suspension must be cooled because the rise in temperature in interaction chamber results in the high operating pressure. Leave the nanoliposomes at temperature above Tc.

\section{Characterization methods}

Size

Measurement of the particle size distribution and polydispersity index of liposomes provides information on their physical stability [23]. Maintaining a constant size and size distribution over a longer time shows liposome stability [24].

\section{Electron microscopy methods}

Electron microscopy methods are used to measure the size of each liposomes with a micron range [23] They may also be used for the visualization of liposomes and for the lamellarity and morphological structure determination. Cryo-TEM could visualize liposomes directly in a frozen state.

\section{Fluorescence microscopy}

Fluorescence microscopy measures the fluorescent liposome size and lamellarity. This process evaluate liposomes at a single level of the particles and can therefore calculate the polydisperse liposome suspensions distribution in size. High sensitivity and ease are among the benefits of fluorescence microscopy.

\section{Atomic force microscopy}

It measures size distribution, morphology, stability, and their aggregation. It also evaluates surface properties and liposomal rigidity. Advantage of AFM is to image the samples in natural environment. It doesn't reflect the true surface topography [25].

\section{Dynamic light-scattering}

It is also known as photon correlation spectroscopy, the dynamic light-scattering technique is mostly used for the established study of liposome size distribution. The dependence of scattered light fluctuations on time is measured by this method [23] The Brownian motion of the colliding particles is measured by DLS, results in the incident's dispersion light [26]. The dispersion of light depends on the difference in the refractive index between the particles suspended and the solvent. The amount of light dispersed is measured and assessed to give the mean particle size of suspended liposomes [26].

\section{Nanoparticle tracking analysis}

Nanoparticle tracking analysis provides a high resolution particle size distribution and concentration analysis of liposomes at a submicron level. It measures particle size diameter from 30-1000 nm [27]. Sample to be analyzed passed through cell which is then illuminated by laser light. It passes through the liquid surface. Refraction occurs in region where liposomes are present and then it can be visualized [28]. The center of liposomes is tracked by software, and relation with that particle size and particle diffusion coefficient is obtained. Both monodisperse and polydisperse suspensions can be measured. Zeta potential can also be measured by detecting their fluorescent signals [29].

\section{Flow cytometry}

Flow cytometry is a technique used to determine the size distribution of liposomes. It is also used to detect the inhomogeneity in their size. It is based on the principle of light scattering [30]. Here the particles are measured in a continuous flow system of closed containment. Liposomes to be fluorescently labeled to distinguish from other impurities [31]. Particle detection performed at $10^{\circ}$ forward angle scatter and $90^{\circ}$ side angle scatter. Optimization is done to detect 1500 particles per second. It can produce reliable and reproducible results.

\section{Size exclusion chromatography}

The liposomal size distribution with size $500 \mathrm{~nm}$ can be measured by size exclusion chromatography. In this method, liposomes can be separated and individually and grouped together depends upon the particle size of the liposomes. Fusion and aggregation characteristics of liposomes can be measured. Composition of chemicals in gels influence the interaction of liposomes and retention depends on shape and size of liposomal composition [32]. 
Porosity of the gel is selective for particles from $100 \mathrm{~nm}$ to several $\mathrm{nm}$. Change in liposomes concentration and composition are due to partition inside the gel column and it causes a defect in quantitative analysis. It can be coupled with high performance liquid chromatography (HPLC). Calibration of column is done to sizing of liposomes [33].

\section{Centrifugal sedimentation}

Size distribution of liposomes with a diameter range of 0.015 to $30 \mathrm{~nm}$ could be measured by Centrifugal sedimentation [15]. Based on principles of hydrodynamic theory, Sedimentation velocity is quadratically proportional to the particle diameter [34]. Time taken by the sample to reach the detector gives the particle size. Sedimentation velocity of the sample is calculated, if fluid density, fluid viscosity and centrifugal acceleration are known [15]. Under stable conditions, particles sediment at the bottom layer and enlightened with light which is used to measure particle size and concentrations.

\section{Differential scanning calorimetry}

Differential scanning calorimetry is a tool to study the thermal transition behavior of liposomes. This method is sensitive to the liposomal physicochemical properties [35]. Two methods are widely used to measure excess heat capacity. The first one uses the heat leak principle and second works by temperature increment of the system at a constant rate and energy needed to attain the same temperature between sample and reference [36]. The plot of heat capacity $v s$ temperature used to determine the transition temperature (Tm). It gives both qualitative and quantitative data of exothermic and endothermic reactions takes place in the sample.

\section{Encapsulation efficiency}

Encapsulation efficiency defines the amount of drug that is encapsulated in the liposomes [37]. The maximum amount of drug could be encapsulated is called encapsulation capacity [38]. It can be defined as the ratio of concentration of drug and concentration of phospholipids:

$\mathrm{EC}=\mathrm{Ce} /(\mathrm{Ci} \times \mathrm{CL})$

where EC - Encapsulation capacity; Ce Concentration of encapsulated drug; $\mathrm{Ci}$ -
Concentration of input drug; CL - Concentration of lipids.

The process starts with removal of free encapsulated drug from liposomal suspension, which can be done by different techniques [39]. Mini-column centrifugation is based on the difference in size of liposomes and free drug. Sample applies to Sephadex gel column and then centrifuged. Liposomes are expelled, free drug gets bound to column. Then, the encapsulation efficiency is calculated by the lipid bilayer destruction and process of quantification [40].

\section{Stability of liposomes}

Liposomal stability includes physical, chemical, and biological means, and they are interrelated. Liposomes shelf life depends on physical characteristics such as size distribution, encapsulation efficiency and chemical characteristics such as phospholipid layer degradation. Physical stability of preparations is difficult to maintain after once planned. Change in size distribution and stability issues because of degradation are major problems in storage. The leakage of encapsulated material is because of membrane permeability.

Addition of antioxidants during manufacturing can prevent oxidation of chemical structure of liposomes. Lyophilization can help in prevention of oxidation and hydrolysis [41]. Lyophilization without stabilizers can lead to fusion of vesicles, and hence, cyclo protectants is used.They protect against the rupture of liposomal membrane.

New methods and formulation parameters are required to be optimized to avoid these types of stability problems [42]. Lyophilization helps to increase shelf life of liposomal formulations. Liposomal AmBisome (Amphotericin) is a first liposomal product supplied as lyophilized powder [43].

\section{Sterilization of liposomes}

The approaches to sterility assurance in parenteral products include final product sterilization in container and aseptic manufacturing. Terminal sterilization is widely used because of high sterility assurance. For liposomal products, UV sterilization, gamma sterilization and filtration are mostly preferred. Because of sensitivity, physical and chemical degradation of liposomes may occur if heat sterilization is used. Filtration sterilization has limitations because of liposomes smaller than 
$200 \mathrm{~nm}$ diameter and longer time periods required for the process which is ineffective for viruses removal $[44,45]$.

\section{Application of liposomes in cancer therapy}

Anticancer drugs have a large cytotoxic effect on cancer cells and exhibits a decreased anti-tumor effect in in-vivo clinical treatment procedures. Low therapeutic index is one of the major drawbacks of anti-cancer drugs, showing that the dosage needed to achieve an effect is toxic to normal tissue cells. This may be due to inability to achieve increased Therapeutic concentration at the targeted sites. It may result from nonspecificity to normal tissues such as GI tract, Cardiac, renal, as well as from inappropriate formulation of drugs [46].

Most of the liposomal formulations of anticancer drugs shows reduced toxicity to that of free drug and find its application in the preclinical stage of cancer therapy [47]. Encapsulation of drugs in liposomes is the reason for increased blood circulation time. Sometimes it may cause an enhanced deposition in tissues and distribution is altered. For cancer cells targeting, liposomes are efficient carrier with minimal toxicity which shows potential to enter transcapillary passage. Studies show that liposomes resides in higher concentration in cancer tissues than normal tissues [43]. Anthracycline class of drugs works by stopping the dividing cells growth by Intercalation into DNA. This drug class is very toxic. Many research works have directed towards increasing the safety profile of these drugs. Encapsulation of drugs results in reduced

\section{REFERENCES}

1. Sercombe L, Veerati T, Moheimani F, Wu SY, Sood AK, Hua S. Advances and challenges of liposome assisted drug delivery. Front Pharmacol. 2015;6:286. doi:10.33 89/fphar.2015.00286

2. Hardiansyah A, Huang LY, Yang MC, et al. Magnetic liposomes for colorectal cancer cells therapy by highfrequency magnetic field treatment. Nanoscale Res Lett. 2014;9(1):497. doi:10.1186/1556-276X-9-497

3. Biehl P, Von der Lühe M, Dutz S, Schacher FH. Synthesis, characterization, and applications of magnetic nanoparticles featuring polyzwitterionic coatings. Polymers (Basel). 2018;10(1):91. doi:10.3390/polym10 010091

4. Nam JH, Kim S-Y, Seong H. Investigation on physicochemical characteristics of a nanoliposomebased system for dual drug delivery. Nanoscale Res Lett. 2018;13(1):101. doi:10.1186/s11671-018-2519-0

5. Flannery MC. The "Liposome" Letters. Am Biol Teach. 1987;49(2):122-124. doi:10.2307/4448454

6. Çağdaş M, Sezer AD, Bucak S. Liposomes as potential drug carrier systems for drug delivery. In: Sezer AD cardiotoxicity, dermal toxicity than free anticancer drugs [47].

US Foods and Drug Administration (FDA) approves two liposomal formulations for commercial use in treatment of Kaposi sarcoma. Doxil (Doxorubicin) is the FDA approved liposomal drug and in market since 1995. After six months, Daunorubicin was approved due to their mechanical stability in their liposomal bilayered structure [47].

\section{CONCLUSION}

Magnetic nanoliposomes are a crucial drug delivery system, suitable for potential use in targeted and controlled release mechanisms. In current years, several important liposomal formulations have been developed for different diseases. The various preparation and characterization techniques have been developed with the advancement of technology. Magnetic nanoliposomes are the effective carriers to enhance therapeutic effectiveness of drugs in terms of duration of action and to optimize the dosage regimen with higher efficacy and reduced toxicity. Magnetic nanoliposomes are the important carriers for therapeutic efficiency in term of duration of action, decrease in dose frequency and delivering drugs at higher efficacy and lower toxicity. Even though the technological advancements have led to development in area of liposome research, there is a need of improved design and stability optimization, in addition to efficient production and characterization.

(ed.). Application of Nanotechnology in Drug Delivery. IntechOpen: London, UK, 2014. doi:10.5772/58459

7. Akbarzadeh A, Rezaei-Sadabady R, Davaran S, et al. Liposome: Classification, preparation, and applications. Nanoscale Res Lett. 2013;8(1):102. doi:10.1186/1556276X-8-102

8. Szoka Jr F, Papahadjopoulos D. Comparative properties and methods of preparation of lipid vesicles (liposomes). Ann Rev Biophys Bioeng. 1980;9(1):467508. doi:10.1146/annurev.bb.09.060180.002343

9. Mozafari MR. Nanoliposomes: Preparation and analysis. Methods Mol Biol. 2010;605:29-50. doi:10.1007/978-160327-360-2_2

10. Shubayev VI, Pisanic II TR, Jin S. Magnetic nanoparticles for theragnostics. Adv Drug Deliv Rev. 2009;61(6):46777. doi:10.1016/j.addr.2009.03.007

11. Berger P, Adelman NB, Beckman KJ, Campbell DJ, Ellis $A B$, Lisensky GC. Preparation and properties of an aqueous ferrofluid. J hem Educ. 1999;76(7):943.

12. Pattni BS, Chupin VV, Torchilin VP. New developments in liposomal drug delivery. Chem Rev. 2015;115(19): 
10938-66. doi:10.1021/acs.chemrev.5b00046

13. Nomani S, Samy JG. Nanoliposome: An alternative approach for drug delivery system. Int J Adv Pharmacy Med Bioallied Sci. 2016(2016):92.

14. Zhang H. Thin-film hydration followed by extrusion method for liposome preparation. Methods Mol Biol. 2017;1522:17-22. doi:10.1007/978-1-4939-6591-5_2

15. Namvari $M$, Namazi $H$. Clicking graphene oxide and $\mathrm{Fe}_{3} \mathrm{O}_{4}$ nanoparticles together: an efficient adsorbent to remove dyes from aqueous solutions. Int J Environ Sci Technol. 2014;11(6):1527-36. doi:10.1007/s13762-01 4-0595-y

16. Filipe V, Hawe A, Jiskoot W. Critical evaluation of nanoparticle tracking analysis (NTA) by NanoSight for the measurement of nanoparticles and protein aggregates. Pharm Res. 2010;27(5):796-810. doi:10.100 7/s11095-010-0073-2

17. Bárcena C, Sra AK, Gao J. Applications of magnetic nanoparticles in biomedicine. In: Liu J, Fullerton E, Gutfleisch O, Sellmyer D. (eds.). Nanoscale magnetic materials and applications. Springer: Boston, MA, 2009; 591-626. doi:10.1007/978-0-387-85600-1_20

18. Rahman A, Uahengo V, Likius D. Mini review on emerging methods of preparation of liposome and its application as Liposome drug delivery systems. Open J Pharmacol Pharmacother. 2018;3(1):5-21. doi:10.1735 2/ojpp.000007

19. Majidi S, Zeinali Sehrig F, Farkhani SM, Soleymani Goloujeh M, Akbarzadeh A. Current methods for synthesis of magnetic nanoparticles. Artif Cells Nanomed Biotechnol. 2016;44(2):722-34. doi:10.3109/21691401. 2014.982802

20. Zylberberg C, Matosevic S. Pharmaceutical liposomal drug delivery: a review of new delivery systems and a look at the regulatory landscape. Drug Deliv. 2016;23(9):3319-29. doi:10.1080/10717544.2016.117 7136

21. Dua JS, Rana AC, Bhandari AK. Liposome: Methods of preparation and applications. Int J Pharm Stud Res. 2012;3(2):14-20.

22. Reza Mozafari M. Liposomes: An overview of manufacturing techniques. Cell Mol Biol Lett. 2005; 10(4):711-9.

23. Szuplewska A, Rękorajska Joniec A, Pocztańska E, Krysiński P, Dybko A, Chudy M. Magnetic field-assisted selective delivery of doxorubicin to cancer cells using magnetoliposomes as drug nanocarriers. Nanotechnology. 2019;30(31):315101. doi:10.1088/13 61-6528/ab19d3

24. Woodle MC, Papahadjopoulos D. Liposome preparation and size characterization. Methods Enzymol. 1989;171: 193-217. doi:10.1016/s0076-6879(89)71012-0

25. Kanásová M, Nesměrák K. Systematic review of liposomes' characterization methods. Monatsh Chem. 2017;148(9):1581-93. doi:10.1007/s00706-017-19949

26. Maja L, Željko K, Mateja P. Sustainable technologies for liposome preparation. J Supercrit Fluids. 2020;165: 104984. doi:10.1016/j.supflu.2020.104984

27. Reshetov V, Zorin V, Siupa A, D'Hallewin MA, Guillemin F, Bezdetnaya L. Interaction of liposomal formulations of meta-tetra (hydroxyphenyl) chlorine (temoporfin) with serum proteins: protein binding and liposome destruction. Photochem Photobiol. 2012;88(5):1256-64. doi:10.1111/j.1751-1097.2012.01176.x

28. Saveyn H, De Baets B, Thas O, Hole P, Smith J, Van der Meeren P. Accurate particle size distribution determination by nanoparticle tracking analysis based on 2-D Brownian dynamics simulation. J Colloid Interface Sci. 2010;352(2):593-600. doi:10.1016/j.jcis. 2010.09.006

29. Chen C, Zhu S, Huang T, Wang S, Yan X. Analytical techniques for single-liposome characterization. Anal Methods. 2013;5(9):2150-7. doi:10.1039/C3AY40219C

30. Salzman GC, Wilder ME, Jett JH. Light scattering with stream-in-air flow systems. J Histochem Cytochem. 1979;27(1):264-7. doi:10.1177/27.1.374583

31. Childers NK, Miller KL, Tony G, Llarena JC, Greenway T, Ulrich JT, Michalek SM. Adjuvant activity of monophosphoryl lipid A for nasal and oral immunization with soluble or liposome-associated antigen. Infect Immun. 2000;68(10):5509-16. doi:10.11 28/IAI.68.10.5509-5516.2000

32. Grabielle-Madelmont C, Lesieur S, Ollivon M. Characterization of loaded liposomes by size exclusion chromatography. J Biochem Biophys Methods. 2003;56(1-3):189-217. doi:10.1016/S0165-022X(03)0 0059-9

33. Lesieur S, Grabielle-Madelmont C, Paternostre M, Ollivon M. Study of size distribution and stability of liposomes by high performance gel exclusion chromatography. Chem Phys Lipids. 1993;64(1-3):57-82. doi:10.1016/0009-3084(93)90058-B

34. Biltonen RL, Lichtenberg D. The use of differential scanning calorimetry as a tool to characterize liposome preparations. Chem Phys Lipids. 1993;64(1-3):129-42. doi:10.1016/0009-3084(93)90062-8

35. Ross PD, Goldberg RN. A scanning microcalorimeter for thermally induced transitions in solution. Thermochim Acta. 1974;10(2):143-51. doi:10.1016/0040-6031(74) 85033-1

36. Privalov PL, Plotnikov VV, Filimonov VV. Precision scanning microcalorimeter for the study of liquids. $J$ Chem Therm. 1975;7(1):41-7. doi:10.1016/0021-9614 (75)90079-8

37. Pattni BS, Chupin VV, Torchilin VP. New developments in liposomal drug delivery. Chem Rev. 2015;115(19): 10938-66. doi:10.1021/acs.chemrev.5b00046

38. Padamwar MN, Pokharkar VB. Development of vitamin loaded topical liposomal formulation using factorial design approach: drug deposition and stability. Int $J$ Pharm. 2006;320(1-2):37-44. doi:10.1016/j.ijpharm.20 06.04.001

39. Benita S, Poly PA, Puisieux F, Delattre J. Radiopaque liposomes: Effect of formulation conditions on encapsulation efficiency. J Pharm Sci.1984;73(12):17515. doi:10.1002/jps.2600731223

40. Lambelet P, Löliger J. The fate of antioxidant radicals during lipid autooxidation. I. The tocopheroxyl radicals. Chem Phys Lipids. 1984;35(3):185-98. doi:10.1016/00 09-3084(84)90045-8

41. Anchordoquy TJ, Carpenter JF, Kroll DJ. Maintenance of transfection rates and physical characterization of lipid/DNA complexes after freeze-drying and rehydration. Arch Biochem Biophy. 1997;348(1):199206. doi:10.1006/abbi.1997.0385 
42. Lasic DD, Čeh B, Stuart MC, Guo L, Frederik PM, Barenholz Y. Transmembrane gradient driven phase transitions within vesicles: lessons for drug delivery. Biochim Biophys Acta. 1995;1239(2):145-56. doi:10.101 6/0005-2736(95)00159-Z

43. Lukyanov AN, Torchilin VP. Autoclaving of liposomes. $J$ Microencapsul. 1994;11(6):669-72. doi:10.3109/02652 049409051118

44. d C Molina M, Armstrong TK, Zhang YE, Patel MM, Lentz YK, Anchordoquy TJ. The stability of lyophilized lipid/DNA complexes during prolonged storage. J Pharm Sci. 2004;93(9):2259-73. doi:10.1002/jps.20138
45. Toh M-R, Chiu GNC. Liposomes as sterile preparations and limitations of sterilisation techniques in liposomal manufacturing. Asian J Pharm Sci. 2013;8(2):88-95.

46. Pradhan P, Giri J, Banerjee R, Bellare J, Bahadur D. Preparation and characterization of manganese ferritebased magnetic liposomes for hyperthermia treatment of cancer. J Magn Magn Mater. 2007;311(1):208-15.

47. Sharma A, Mayhew E, Block L, Cavanaugh C, Harmon P, Janoff A, Bernacki RJ. Activity of paclitaxel liposome formulations against human ovarian tumor xenografts. Int J Cancer. 1997;71(1):103-7. doi:10.1002/(sici)10970215(19970328)71:1<103::aid-ijc17>3.0.c0;2-j 